\title{
Solo, TOPOGRAFIA, MORTALIDADE E RECRUTAMENTO NA FLORESTA TROPICAL ESTACIONAL DECIDUAL
}

\author{
Andre Eduardo Gusson ${ }^{1}$, Ana Paula de Oliveira ${ }^{2}$, Vagner Santiago do Vale ${ }^{3}$, Glein Monteiro de \\ Araújo $^{4}$, Ivan Schiavini ${ }^{4}$
}

${ }^{1}$ Docente do Instituto de Ensino Superior ILES/ULBRA

${ }^{2}$ Universidade Federal de Goiás

${ }^{3}$ Univerisdade Estadual de Goiás

${ }^{4}$ Instituto de Biologia, Universidade Federal de Uberlândia

Recebido em 06 de outubro de 2017. Aceito em 30 de março de 2018. Publicado em 06 de abril de 2018.

\begin{abstract}
Resumo - Muitos são os fatores que podem aumentar ou diminuir a intensidade da dinâmica nas florestas tropicais. Com certeza o clima pode ser considerado fator determinante para as taxas de mortalidade e recrutamento de árvores nestas florestas. Entretanto, a topografia e o tipo de solo também são fatores que sinergicamente influenciam a distribuição e composição das espécies arbóreas, consequentemente alterando as taxas de dinâmica, pois algumas espécies são mais sensíveis às mudanças no ambiente. O objetivo deste trabalho foi avaliar a dinâmica de uma floresta tropical decidual situada sobre um gradiente edáfico e topográfico, juntamente com a interpretação das variações pluviométricas registradas no intervalo do estudo. Os resultados revelam que a floresta apresenta uma dinâmica menos intensa, com taxas de mortalidade e recrutamento abaixo do esperado paras as floresta tropicais deciduais sob forte sazonalidade, assemelhandose as taxas das florestas pluviais tropicais. A relação do grupo de espécies arbóreas com o tipo de solo e topografia e uma possível estabilidade pluviométrica podem interferir e direcionar os fatores que determinam os resultados das taxas de mortalidade e recrutamento em estudos de dinâmica nas florestas tropicais.
\end{abstract}

Palavras-chave: Rotatividade; Floresta Estacional; Unidade De Conservação.

\section{SOIL, TOPOGRAPHY, MORTALITY AND RECRUITMENT IN SEASONAL DECIDUOUS TROPICAL FORESTS}

Abstract - There are many factors that can increase or decrease the dynamics intensity in tropical forests. Certainly, the climate can be considered a determining factor for tree mortality and recruitment rates in these forests. However, topography and soil are factors that synergistically influence the distribution and the composition of tree species, consequently altering the dynamic rates, since some tree species are more sensitive to changes in the environment. The objective of this study was to evaluate the dynamics in deciduous tropical forest located on an edaphic and topographic gradient, as well as to interpret the rainfall variations observed during the research. The results show that the forest has a less intense dynamics, with mortality rates and recruitment below the rates one would expect in a seasonal deciduous tropical forests, resembling the rates of a tropical rainforest. The relationship between a group of tree species and soil and topography type and a possible rainfall stability can interfere and direct the factors that determine the results of the mortality and recruitment rates in dynamic studies of tropical forests.

Keywords: Turnoter; SEASONAL ForEst; CONSERVATION UNIT. 
REsumen - Muchos factores pueden aumentar o disminuir la intensidad de la dinámica de los bosques tropicales. Ciertamente, el clima puede ser considerado como un factor determinante para las tasas de mortalidad y reclutamiento de árboles en estos bosques. Sin embargo, la topografía y el tipo de suelo son también factores que influyen de forma sinérgica la distribución y composición de las especies arbóreas, consecuentemente alterando las tasas de dinámica, pues algunas especies son más sensibles a los cambios en el ambiente. El objetivo de este trabajo fue evaluar la dinámica de un bosque tropical deciduo ubicado sobre un gradiente edáfico y topográfico, juntamente con la interpretación de las variaciones en las precipitaciones registradas en el tiempo de estudio. Los resultados revelan que el bosque presenta una dinámica menos intensa, con tasas de mortalidad y reclutamiento debajo de lo esperado para los bosques tropicales deciduos altamente estacionales, donde sus tasas se asemejan a las tasas de las selvas tropicales. La relación entre el grupo de especies arbóreas con el tipo de suelo y topografía y una posible estabilidad de precipitaciones pueden interferir y direccionar los factores que determinan los resultados de las tasas de mortalidad y reclutamiento en estudios de dinámica en los bosques tropicales.

Palabras clave: Rotación; Bosque Estacional; Zonas De Conservación.

\section{INTRODUÇÃO}

As informações históricas sobre o sistema de uso das terras tropicais revelam que a maioria das florestas contínuas está inserida em um mosaico com campos de agricultura e fragmentos de florestas em diferentes níveis de sucessão (Quesada et al. 2009).

$\mathrm{Na}$ América do Sul, a situação destas florestas é particularmente ameaçadora, principalmente para a floresta tropical sazonalmente secas, que concentra grande parte da distribuição neste continente (Miles et al. 2006). A floresta tropical decidual pode ser definida como um sistema de florestas sob a influencia da sazonalidade climática com severos meses de seca durante um período do ano (Pennigton et al. 2009). No Brasil, estudos revelam que a floresta tropical sazonalmente seca ou floresta estacional decidual apresenta uma dinâmica da vegetação mais intensa quando comparado com outras formações florestais (Swaine et al. 1990; Gijsbers et al. 1994; Venkateswaran e Parthasarathy 2005; Carvalho e Felfili 2011; Lévesque et al. 2011).

No Cerrado ocorre a marcante dominância da floresta estacional decidual proporcionada pela sazonalidade climática do Bioma, caracterizado por um período de déficit hídrico entre os meses de abril e setembro. Esta sazonalidade climática conduz mudanças na composição e estrutura das florestas (Quigley e Platt 2003) e os estudos de dinâmica vegetal demonstram estas mudanças por análises de parâmetros na comunidade arbórea, principalmente mortalidade e recrutamento (Werneck et al. 2000; Werneck e Franceschinelli 2004).

Recentes estudos demonstram que períodos mais intensos de seca podem alterar a composição, a estrutura e a biogeografia das florestas (Allen et al. 2010; Espinosa et al. 2011). Outros estudos demonstram os efeitos das condições edáficas sobre as variáveis de dinâmica vegetal (Madelaine et al. 2007; Higuchi et al. 2008), a influencia da topografia nas características químicas do solo (Griffiths et al. 2009) e como a variação edáfica afeta a distribuição e ocorrência das espécies vegetais (Souza et al. 2007).

A floresta estacional decidual pode apresentar variações na composição da comunidade arbórea em unidades relativamente próximas, criando comunidades vegetais distintas numa mesma floresta (Souza et al. 2007). Nesse sentido, questionamos como seria a dinâmica nestas distintas comunidades vegetais; será que as variáveis ambientais poderiam interferir nos parâmetros de recrutamento e mortalidade das espécies vegetais e criar um moisaco florestal? Assim, acreditamos que as diferenças na dinâmica das comunidades arbóreas sejam tão distintas quanto às espécies que as compõem. Baseado nestas indagações o estudo tem com objetivo avaliar a 
dinâmica da floresta tropical decidual sobre diferentes condições edáficas, topográficas e relacionar a intensidade dos parâmetros de mortalidade e recrutamento com a variação das condições pluviométricas.

\section{MATERIAL E MÉTODOS}

\section{Caracterização da área de estudo}

O estudo foi conduzido em uma floresta tropical decidual no Parque Estadual do Pau Furado (PEPF) localizado no município de Uberlândia, Minas Gerais (MG), Brasil (Figura 1). O PEPF apresenta 2,2 mil hectares de vegetação protegida com domínio da floresta estacional decidual submontana (IBGE 2012). A área de estudo possui cerca de 70 hectares e situa-se nas coordenadas $18^{\circ} 48^{\prime} 07^{\prime \prime S}$ e $48^{\circ} 10^{\prime} 24^{\prime \prime} \mathrm{O}$. A floresta esta localizada em uma encosta com forte declividade, distribuída em gradiente edáfico, constituído por rochas de origem no basalto e micaxisto (Souza et al. 2007).

O clima local é do tipo Aw, megatérmico, com chuvas no verão e seca no inverno, segundo a classificação de Köppen. De acordo com Alvares et al. (2014), a temperatura média anual é de $22^{\circ} \mathrm{C}$ e pluviosidade em torno de $1550 \mathrm{~mm}$ /ano.

Figura 1 - Localização geográfica da área de estudo no Parque Estadual do Pau Furado, Uberlândia, Minas Gerais, Brasil.

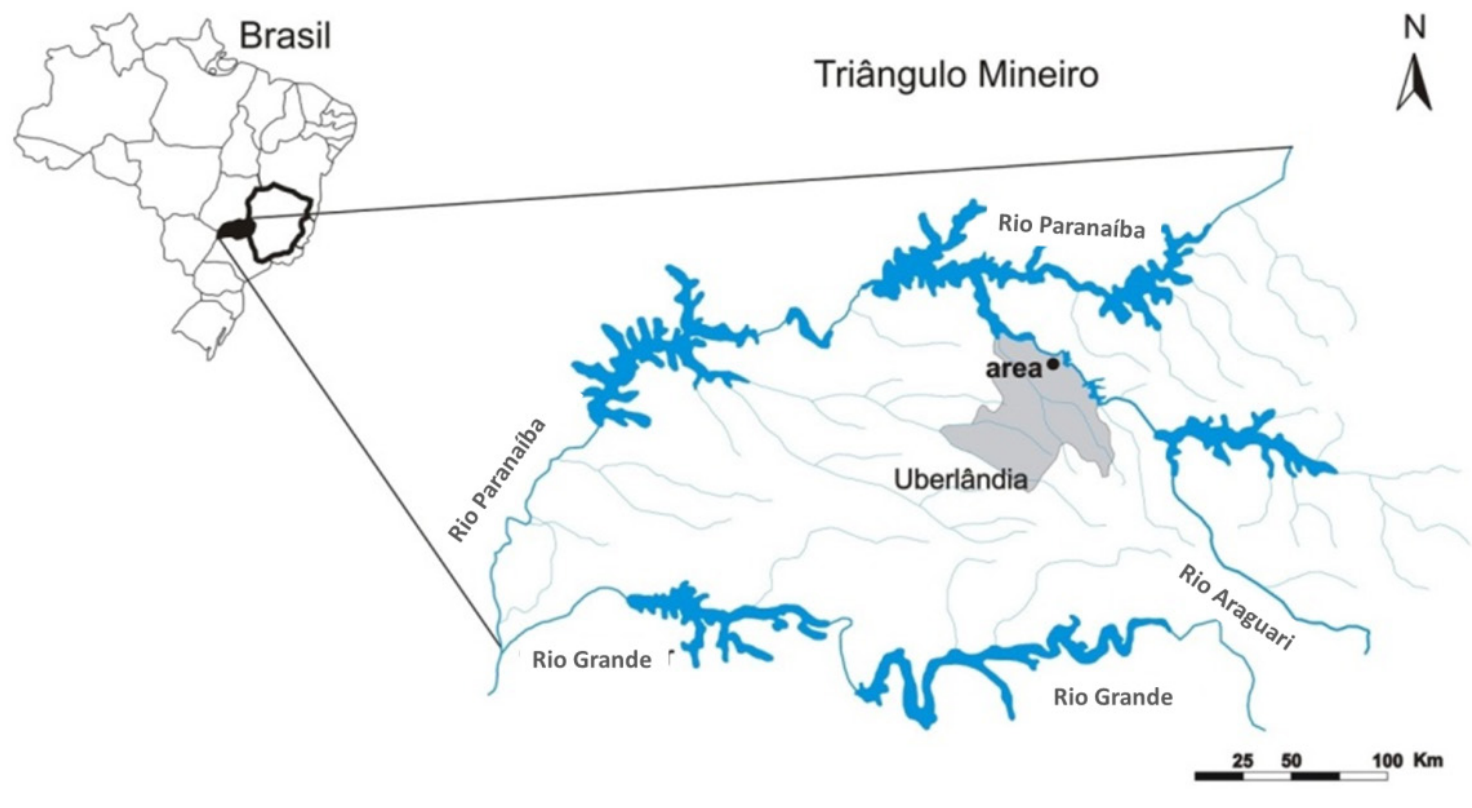

\section{Coleta de dados}

O primeiro estudo na comunidade arbórea foi conduzido por Souza et al (2007) no ano de $2004\left(\mathrm{~T}_{0}\right)$. A área amostral compreende um hectare, distribuído em 50 parcelas de 10 x 20 metros, alocadas num gradiente edáfico e topográfico. O estudo seguiu o protocolo de parcelas permanentes e o critério de inclusão adotado foi diâmetro a altura do peito (DAP, 1,30m acima do nível do solo) maior ou igual a 4,77 cm. O segundo estudo foi realizado no ano de $2010\left(\mathrm{~T}_{1}\right)$ com nova amostragem da comunidade arbórea utilizando o mesmo critério de 
amostragem. Os indivíduos mortos foram registrados e os indivíduos recrutas, ou seja, aqueles que atingiram o limite de inclusão de $\mathrm{DAP} \geq 4,77 \mathrm{~cm}$ no $\mathrm{T}_{1}$ também foram registrados e medidos.

Os dados de pluviosidade foram obtidos a partir do banco de dados da estação meteorológica do Laboratório de Climatologia e Recursos Hídricos, localizada no município de Uberlândia, Minas Gerais, para um período de 28 anos.

\section{Análise dos dados}

As taxas de dinâmica da floresta foram calculadas para a amostra total da comunidade arbórea e para os grupos de amostras nas condições edáficas (basalto e micaxisto) e topográfica (declividade forte e extra forte). As taxas anuais de mortalidade $(M)$ e recrutamento $(R)$ foram calculadas através do modelo proposto por Sheil e May (1996), adaptado por Oliveira-Filho et al. (2007).

As variáveis matemáticas acima citadas expressam a dinâmica da comunidade arbórea em termos de densidade e da área basal, revelando as taxas anuais de mortalidade $(M)$, recrutamento $(R)$, perda $(P)$ e ganho $(G)$ como sugerido por Oliveira-Filho et al. (2007), as taxas de rotatividade $\left(T_{N}\right)$ como aplicado por Oliveira-Filho et al. (1997) e Werneck e Franceschinelli (2004) e as taxas de mudança líquida $\left(C h_{N}\right)$ conforme Korning e Balslev (1994). As equações utilizadas seguem os seguintes modelos matemáticos:

$$
\begin{array}{ll}
M=\left\{1-\left[\left(N_{0}-m\right) / N_{0}\right]^{1 / t}\right\} \times 100 & T_{\mathrm{N}}=(M+\mathrm{R}) / 2 \\
\mathrm{R}=\left[1-\left(1-r / N_{t}\right)^{1 / \mathrm{t}}\right] \times 100 & T_{\mathrm{AB}}=(P+G) / 2 \\
P=\left\{1-\left[\left(A B_{0}-A B_{m}+A B_{d}\right) / A B_{0}\right]^{1 / \mathrm{t}}\right\} \times 100 & C h_{\mathrm{N}}=\left[(\mathrm{N} t / \mathrm{N} 0)^{1 / \mathrm{t}-1}\right] \times 100 \\
G=\left\{1-\left[1-\left(A B_{r}+A B_{g} / A B_{t}\right]^{1 / \mathrm{t}}\right\} \times 100\right. & C h_{\mathrm{N}}=\left[\left(A B_{t} / A B_{0}\right)^{1 / \mathrm{t}}-1\right] \times 100
\end{array}
$$

Onde, $\mathrm{t}=$ intervalo de tempo entre as medições, $N_{0}$ e $N_{\mathrm{t}}=$ respectivamente, o número de indivíduos inicial e final, $m$ e $r=$ respectivamente, o número de indivíduos mortos e recrutados, $A B_{0}$ e $A B_{\mathrm{t}}=$ respectivamente, as áreas basais inicial e final das árvores, $A B_{\mathrm{m}} \mathrm{e} A B_{\mathrm{r}}=$ respectivamente, a área basal dos indivíduos mortos e dos recrutas e $A B_{\mathrm{d}}$ e $A B_{\mathrm{g}}=$ respectivamente, o decremento e incremento em área basal das árvores sobreviventes.

Os principais parâmetros fitossociológicos (riqueza de espécies, índice de diversidade, densidade $-\mathrm{n}^{\circ}$ de indivíduos e dominância - área basal) foram comparados entre os anos utilizando o teste $t$ pareado. $O$ teste $t$ foi aplicado para aferir as diferenças de taxas de dinâmica nas diferentes condições edáficas (basalto vs. micaxisto) e topográfica (forte vs. extra forte) da floresta. Uma análise de correspondência (CA) foi aplicada para verificar em qual condição edáfica e topográfica as taxas de dinâmica foram superiores. Uma análise de variância (ANOVA) foi aplicada aos dados de pluviosidade para demonstrar as oscilações na média pluviométrica e os períodos de déficit hídrico no intervalo de tempo entre 1981 e 2009, qual abrange o período de estudo.

O índice de diversidade de Shannon-Wienner $\left(H^{\prime}\right)$ foi calculado utilizando o FITOPAC-SHELL 1.6 (Shepherd 2006), seguido do teste de Hutcheson (1970) aplicado para verificar se houve diferença significativa entre os índices de $H^{\prime}$ obtidos para o período de intervalo do estudo (Magurran 2004). Todas as análises estatísticas foram realizadas com o programa SYSTAT (Wilkinson 2002), utilizando um intervalo de confiança de $95 \%$ e seguindo os critérios de homogeneidade e normalidade dos dados. 


\section{Resultados}

Os resultados fitossociológicos demonstram resultados favoráveis e significativos para a floresta, com aumento do número de indivíduos, espécies e incremento na área basal das árvores. A tabela 1 demonstra os principais parâmetros fitossociológicos e os valores das diferenças para o tempo do estudo.

Tabela 1 - Principais parâmetros fitossociológicos da comunidade arbórea na floresta tropical decidual localizada no PEPF, Uberlândia, Minas Gerias, Brasil.

\begin{tabular}{llll}
\hline Parâmetros & $\mathbf{2 0 0 4}\left(\mathbf{T}_{\mathbf{0}}\right)$ & $\mathbf{2 0 1 0}\left(\mathbf{T}_{\mathbf{1}}\right)$ & Diferença \\
\hline Número de indivíduos & 1064 & 1111 & $p=0.014$ \\
Número de espécies & 57 & 70 & $p=0.019$ \\
Índice de Shannon-Wienner $\left(H^{\prime}\right)$ & 2,77 & 2,92 & $p>0.100$ \\
Equabilidade $(J)$ & 0,68 & 0,68 & $p>0.100$ \\
Área basal/hectare & 15,379 & 17,476 & $p<0.001$ \\
\hline
\end{tabular}

A taxa de mortalidade para as árvores na floresta foi de $1,53 \%$ ano $^{-1}$. O recrutamento foi superior à mortalidade $(t=2,246, g l=49, p=0,029)$ com valor de $1,95 \% \mathrm{ano}^{-1}$. De uma forma geral, a floresta demostra um aumento líquido na densidade e na área basal das espécies vegetais na comunidade arbórea em ambas as condições, edáficas e topográficas. Este aumento é resultado de elevadas taxas de recrutamento das espécies e ganho em área basal dos indivíduos. Abaixo, na tabela 2, são apresentadas as principais taxas de dinâmica para a floresta.

Tabela 2 - Principais parâmetros indicadores da dinâmica vegetal da comunidade arbórea na floresta tropical decidual, localizada no PEPF, Uberlândia, Minas Gerais, Brasil.

\begin{tabular}{lccccc}
\hline Amostragem: & Total & $\begin{array}{c}\text { Basalto } \\
\text { Solo }\end{array}$ & $\begin{array}{c}\text { Micaxisto } \\
\text { Dorte } \\
\text { Declividade }\end{array}$ & $\begin{array}{c}\text { Muito forte } \\
\text { Número de parcelas }\end{array}$ & \multicolumn{2}{c}{23} & 27 & 19 & 31 \\
Número de árvores: & 50 & & & & \\
Inicial (2004) & 1064 & 539 & 525 & 384 & 680 \\
Final (2010) & 1111 & 543 & 568 & 423 & 688 \\
Sobreviventes & 970 & 487 & 483 & 360 & 610 \\
Mortas & 94 & 52 & 42 & 24 & 70 \\
Recrutas & 143 & 58 & 85 & 65 & 78 \\
Taxa de mortalidade (\% ano-1) & 1,53 & 1,71 & 1,41 & 1,12 & 1,82 \\
Taxa de recrutamento (\% ano-1) & 1,95 & 1,80 & 2,61 & 2,62 & 1,96 \\
Taxa de rotatividade (\% ano-1) & 1,74 & 1,75 & 2,01 & 1,86 & 1,89 \\
Taxa de mudança (\% ano-1) & 0,43 & 0,09 & 1,23 & 1,54 & 0,14 \\
Área basal: & & & & & \\
Inicial $(2004)$ & 15,39 & 7,55 & 7,84 & 5,76 & 9,63 \\
Final $(2010)$ & 17,04 & 8,40 & 8,64 & 6,33 & 10,71 \\
Mortas (m $\left.{ }^{2}\right)$ & 0,85 & 0,66 & 0,19 & 0,14 & 0,71 \\
Decremento dos sobreviventes $\left(\mathrm{m}^{2}\right)$ & $-0,59$ & $-0,25$ & $-0,34$ & $-0,35$ & $-0,24$ \\
Recrutas (m ${ }^{2}$ ) & 0,39 & 0,16 & 0,23 & 0,17 & 0,22
\end{tabular}




\begin{tabular}{llllll} 
Incremento dos sobreviventes $\left(\mathrm{m}^{2}\right)$ & 3,07 & 1,60 & 1,47 & 1,09 & 1,98 \\
Taxa de perdas (\% ano-1) & 1,64 & 2,12 & 1,16 & 1,57 & 1,70 \\
Taxa de ganhos (\% ano- 1$)$ & 3,73 & 3,82 & 3,59 & 3,63 & 3,75 \\
Taxa de rotatividade (\% an0-1) & 2,68 & 2,97 & 2,37 & 2,60 & 2,73 \\
Taxa de mudança (\% ano- 1$)$ & 1,73 & 1,77 & 1,68 & 2,68 & 1,89 \\
\hline
\end{tabular}

Foram registradas diferenças para a taxa de mortalidade, superior nas parcelas com declividade extra forte $(t=2,147, g l=48, p=0,037)$, para a taxa de mudança líquida em número de indivíduos, superior nas parcelas com declividade forte $(t=-2,202, \mathrm{gl}=48, p=0,032)$ e marginalmente significante para a taxa de perda $(t=$ $-1,923, \mathrm{gl}=48, p=0,060)$, superior no grupo de parcelas sobre basalto. Para melhor analisar estes resultados, a análise exploratória CA permitiu visualizar a separação de dois grupos. O primeiro grupo (G1) concentra as taxas de mortalidade e perda nas parcelas com declividade extra forte, localizadas no solo de origem no basalto, enquanto que o segundo grupo (G2) demonstrou concentração das taxas de recrutamento e ganho nas parcelas com declividade forte, no solo de origem no micaxisto (Figura 2).

Figura 2 - Distribuição das parcelas referidas em relação às taxas de moralidade (T.M), recrutamento (T.R), perda e ganho para a comunidade arbórea do fragmento de $\mathrm{FED}$. $\mathrm{BEF}=$ basalto extraforte, $\mathrm{BF}=$ basalto forte, $\mathrm{MEF}=$ micaxisto extraforte e MF = micaxisto forte.

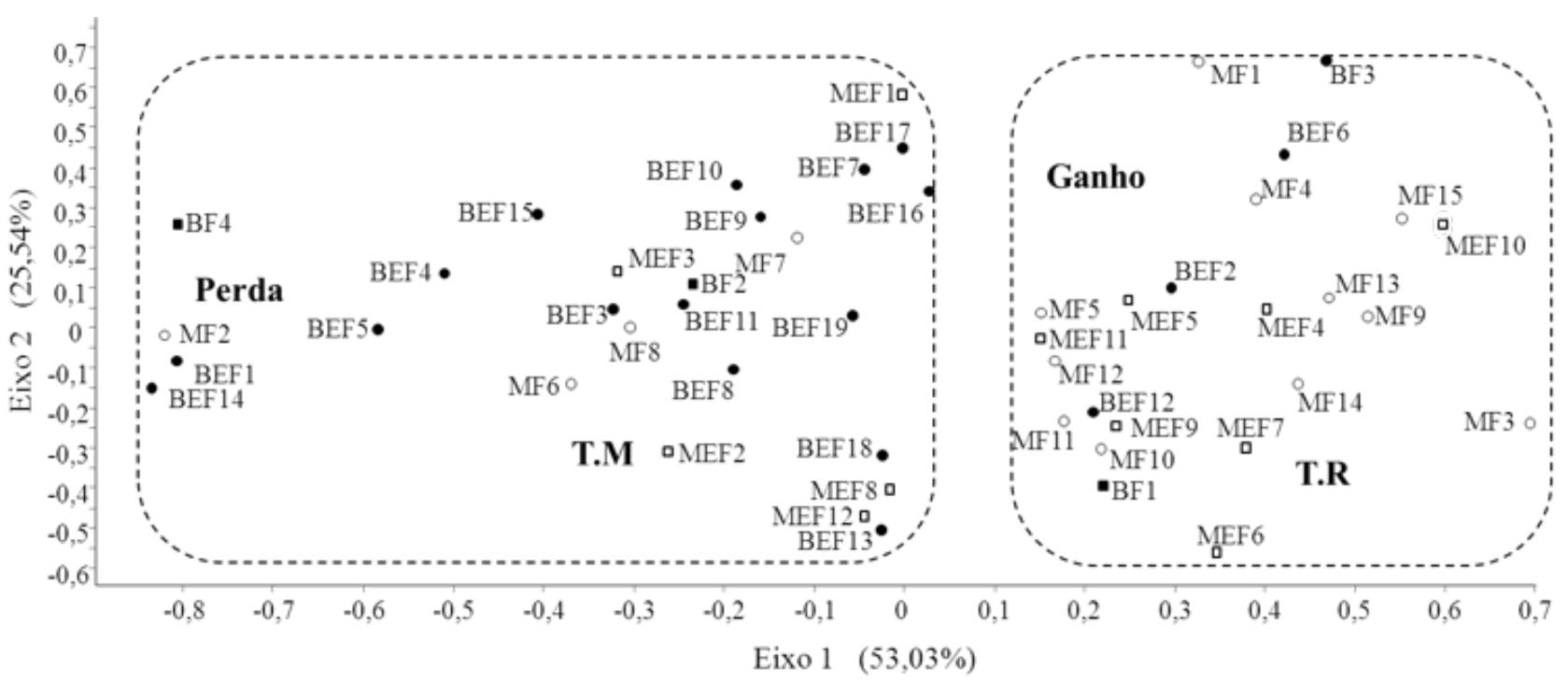

A pluviosidade média anual entre os anos de 1981 a 2009 foi de $1.584 \mathrm{~mm}$, apresentando três anos com maior déficit hídrico, sendo o ano de 1990 o mais rigoroso, com uma pluviosidade média anual de $1.012 \mathrm{~mm}$, seguido pelos anos de 1984 com média de $1.278 \mathrm{~mm}$ e 1999 com média de $1.284 \mathrm{~mm}$. Não houve diferencia para a média de pluviosidade $\left(\mathrm{F}_{5,62}=0,262 p=0,932\right)$ durante o intervalo de tempo do estudo (2004-2010), demonstrando uma condição pluviométrica estável neste período. $\mathrm{Na}$ verdade, as análises demonstraram que, desde 1991, as médias pluviométricas não são diferentes, desconsiderando o déficit hídrico de 1999. Em particular, para a estação chuvosa, considerada o principal período responsável por mudanças na estrutura e dinâmica da vegetação, a pluviosidade diferenciou significativamente apenas para o ano de maior déficit hídrico, $1990(t=-3,290, g l=10 p=0,008)$, revelando períodos de estiagem dentro da estação chuvosa. Assim, os resultados sugerem que a floresta não experimenta uma condição extrema de estresse hídrico para um período de aproximadamente 19 anos. 


\section{Discussão}

O índice de Shannon obtido na comunidade lenhosa da floresta estacional decidual deste estudo registra valores de diversidade próximos ao esperado para esta fitofisionomia (Sagar e Singh 2005; Hérnandez-Ramírez e Garcia-Méndez 2015; Leyser et al. 2012; Silva et al. 2014). Mas, a distribuição da diversidade de espécies na floresta estacional decidual pode ser afetada pelos filtros ambientais (Williams-Linera e Lorea 2009) que estão relacionados com as características do ambiente, a estrutura espacial dos indivíduos e a trajetória sucessional da floresta (Castellanos-Castro e Newton 2015). Antes da instalação do Parque Estadual do Pau Furado, alguns trechos da floresta foram usados como áreas de pastagem ou refúgio para o gado na estação seca. Sabe-se que tempo e a densidade de estocagem de gado na floresta alteram a composição e o percentual de indivíduos no estrato de regeneração da floresta, modificando a diversidade de espécies e consequentemente a dinâmica na floresta (Marquadrdt et al. 2009).

Dados compilados para as florestas tropicais deciduais sujeitas a sazonalidade climática anual revelam uma dinâmica mais intensa, com valores de taxas de mortalidade e recrutamento das comunidades próximas ou superiores a 3\% ao ano (Carvalho e Felfili 2011).

As taxas de mortalidade, recrutamento e rotatividade registradas neste estudo seguem o oposto apresentado pela literatura atual, exibindo taxas muito mais próximas das taxas encontradas para as florestas úmidas, as quais não ultrapassam 2\% ao ano. As taxas de dinâmica da floresta estudada são as mais baixas comparadas a outras florestas tropicais deciduais (Swaine et al. 1990; Sukumar et al. 1992; Werneck e Franceschinelli 2004; Marin et al. 2005; Carvalho e Felfili 2011). Uma explicação para esta aparente diferença nos valores das taxas pode estar vinculado ao critério de inclusão utilizado nos estudos já realizados com o grupo das florestas estacionais deciduais.

A maioria dos estudos de dinâmica utiliza um critério de inclusão com DAP $\geq 10 \mathrm{~cm}$, superior ao padronizado para este estudo (DAP $\geq 4,77 \mathrm{~cm}$ ). O uso de um critério de inclusão maior pode alterar as taxas de dinâmica e prejudicar a interpretação dos resultados (Felfili et al. 2005). Em análise com o mesmo conjunto de dados simulada com DAP $\geq 10 \mathrm{~cm}$ o resultado foi uma forte redução dos valores para as taxas de mortalidade, recrutamento e rotatividade $\left(1,0 \% \mathrm{ano}^{-1}, 0,1 \% \mathrm{ano}^{-1}\right.$ e $0,8 \% \mathrm{ano}^{-1}$, respectivamente), demonstrando estar entre os menores valores para as taxas de mortalidade, recrutamento e rotatividade encontrados em florestas tropicais (Phillips et al. 2004), diferente do esperado para a floresta tropical decidual. Este mesmo padrão de redução nos valores das taxas de mortalidade, recrutamento e rotatividade com o aumento do critério de inclusão foi apresentado em outro estudo realizado por Werneck e Franceschinelli (2004).

As condições abióticas também podem ser consideradas fatores responsáveis pelas diferenças atribuídas às variáveis de dinâmica por determinarem a distribuição de grupos específicos de espécies. Alguns estudos demonstram a forte ação sinérgica que estes fatores abióticos podem ter sobre a dinâmica das comunidades florestais, por exemplo, como a topografia altera a química do solo (Griffiths et al. 2009) e como o solo altera a distribuição e dinâmica das espécies criando comunidades arbóreas distintas em uma unidade florestal (OliveiraFilho et al. 1998; Souza et al. 2007; Madelaine et al. 2007; Higuchi et al. 2008). A concentração da taxa de mortalidade e perda nas áreas com declividade extra forte e basalto pode ser explicada, primeiro, porque as parcelas de basalto coincidem com as parcelas de extra forte declividade, e segundo, a maioria dos indivíduos nestas unidades amostrais pertencem ao grupo de espécies pioneiras. A maioria das plantas pioneiras apresenta 
um ciclo de vida curto e elevada taxa de mortalidade, o que interfere os resultados da dinâmica na floresta (Pooter et al. 2006). Neste ponto de vista, os solos e a topografia influenciam sinergicamente as taxas de dinâmica, entretanto, observamos que o solo é o fator determinante da dinâmica, uma vez que este influencia o grupo de espécies que determinarão os valores para a as taxas de mortalidade e recrutamento de árvores na floresta.

As características ecológicas das espécies são relevantes e ajudam a interpretar os valores atribuídos às taxas de dinâmica. No entanto, as condições ambientais destas florestas podem influenciar estes valores. Os resultados das taxas de dinâmica poderiam estar vinculados não apenas aos fatores já mencionados, como diferenças no critério de inclusão, no tamanho e distribuição das áreas amostrais, no método de cálculo das taxas de dinâmica e no histórico de perturbação da área (Felfili et al. 2005), mas também ser dependente das condições pluviométricas ou histórico pluviométrico do local. As condições pluviométricas durante o tempo do estudo e desde 1991 não flutuaram radicalmente, sugerindo que a floresta experimenta uma situação sem grandes oscilações na disponibilidade hídrica, diferente do que ocorreu no ano de 1990 - anterior ao primeiro levantamento $\left(\mathrm{T}_{0}\right)$ - considerado um ano atípico, com uma baixa disponibilidade hídrica. Os padrões e a variabilidade na precipitação, talvez mais que outros fatores climáticos, são destaque sobre os aspectos que influenciam os processos ecológicos e dinâmicos das florestas tropicais deciduais (Holmgren et al. 2006; Suresh et al. 2010). Um período longo de estiagem pode promover o aumento das taxas de mortalidade (Laurance et al. 2001; Borchert et al. 2002), mesmo para as florestas sujeitas a sazonalidade climática, que são presumivelmente adaptadas ao déficit hídrico durante um período do ano (Suresh et al. 2010). Assim, seria esperado que no ano de 1990, ou em um período próximo, após este momento de maior estresse hídrico, que as taxas de mortalidade seriam maiores que nos anos posteriores. Um novo estudo de dinâmica nesta mesma floresta, possivelmente registraria diferentes taxas, que poderiam ser associadas aos períodos de déficit hídrico e não apenas a variação de espécies e ambientais, evidenciando a importância de incorporar análises pluviométricas para os estudos de dinâmica.

\section{CoNCLUSÕES}

As reduzidas taxas encontradas no presente estudo tanto para a floresta estacional decidual como para as distintas comunidades arbóreas no gradiente edáfico sugerem que a floresta apresenta uma característica peculiar, exibindo uma dinâmica menos intensa no período estudado. A variação edáfica explica a ocorrência das distintas comunidades formadas por grupos de espécies que apresentam estratégias de vida diferenciada, e consequentemente, provoca mudanças nos valores dos principais parâmetros da dinâmica em cada comunidade arbórea existente. Por fim, consideramos que como o presente estudo foi realizado em um período estável para as condições pluviométricas, a dinâmica menos intensa na floresta possa ser um resultado deste período sem grandes oscilações pluviométricas e ainda torna-se importante incorporar as análises pluviométricas para entender se as taxas de dinâmica é um reflexo da comunidade ou da estabilidade climática oferecida, uma vez que, as extremas variações no período de chuva antes e durante um estudo podem alterar as respostas das espécies vegetais e provocar variações nas taxas de dinâmica. 


\section{REFERÊNCIAS}

Allen CD, Macalady AK, Chenchouni H, Bachelet D, McDowell N, Vennetier M, Kitzberger T, Rigling A, Breshears DD, Hogg EH, Gonzalez P, Fensham R, Zhang Z, Castro J, Demidova N, Lim J H, Allard G, Running SW, Semerci A, Cobb N. 2010. A global overview of drought and heat-induced tree mortality reveals emerging climate change risks for forests. Forest Ecology and Management, 259(4):660-684.

Alvares CA, Stape JL, Sentelhas PC, Gonçalves JLM, Sparovek G. 2014. Köppen’s climate classification map for Brazil. Meteorologische Zeitschrift, 22(6):711-728.

Borchert R, Rivera G, Hagnauer W. 2002. Modification of vegetative phenology in a tropical semi-deciduous forest by abnormal drought and rain. Biotropica, 34(1):27-39.

Carvalho FA, Felfili JM. 2011. Variações temporais na comunidade arbórea de uma floresta decidual sobre afloramentos calcários no Brasil Central: composição, estrutura e diversidade florística. Acta Botânica Brasílica, 25(1):203-214.

Catellanos-Castro C, Newton AC. 2015. Environmental heterogeneity influences successional trajectories in Colombian Seasonally Dry Tropical Forests. Biotropica, 47(6):660-671.

Espinosa CI, Cabrera O. 2011. What factors affect diversity and species composition of endangered Tumbesian Dry Forest in Southern Ecuador? Biotropica, 43(1):15-22.

Felfili JM, Carvalho FA, Haidar RF. 2005. Manual de monitoramento de parcelas permanentes nos biomas Cerrado e Pantanal. Brasília: Distrito Federal, 55 p.

Gijsbers HJM, Kessler JJ, Knevel MK. 1994. Dynamics and natural regeneration of woody species in farmed parklands in the Sahel region (Province of Passore, Burkina Faso). Forest Ecology and Management, 64:112.

Griffiths RP, Madritch MD, Swanson AK. 2009. The effects of topography on forest soil characteristics in the Oregon Cascade Mountains (USA): Implications for the effects of climate change on soil properties. Forest Ecology and Management, 257(1):1-7.

Hernández-Ramírez AM, Garcia-Médez S. 2015. Diversidad, estructura y regeneración de la selva tropical estacionalmente seca de la Península de Yucatám, México. International Journal of Tropical Biology, 63(3):603-615.

Higuchi P, Oliveira-Filho AT, Silva AC, Machado ELM, Santos RM, Pifano DS. 2008. Dinâmica da comunidade arbórea em um fragemento de floresta estacional semidecidual montana em Lavras, Minas Geirais, em diferentes classes de solos. Revista Árvore, 32(3):417-423.

Holmgren M, Stapp P, Dickman CR, Gracia C, Graham S, Gutiérrez JR, Hice C, Jaksic F, Kelt DA, Letnic M, Lima M, López BC, Peter L, Meserve PL, Milstead WB, Polis GA, Previtali AM, Richter M, Sabaté S, Squeo FA. 2006. Extreme climatic events shape arid and semiarid ecosystems. Frontiers in Ecology and Evironment, 
$4(2): 87-95$.

Hutcheson K. 1970. A test for comparing diversities based on the shannon formula. Journal of Theoretical Biology, 29(1):151-154

Korning J, Balslev H. 1994. Growth and mortality of trees in Amazonian tropical rain-forest in Ecuador. Journal of Vegetation Science, 5(1):77-86.

Instituto Brasileiro de Geografia e Estatística, 2012. Rio de Janeiro, Brasil. Manual Técnico da Vegetação Brasileira, 271 p.

Laurance, WF, Williamson GB, Delamonica P, Oliveira A, Lovejoy TE, Gascon C, Pohl L. 2001. Effects of a strong drought on Amazonian forest fragments and edges. Journal of Tropical Ecology, 17(6):771-785.

Leyser, G.; Zanin, EM.; Budke, JC.; Mélo, MA.; Henke-Oliveira, C. 2012. Regeneração de espécies arbóreas e relações com componente adulto em uma floresta estacional no vale do rio Uruguai, Brasil. Acta Botanica Brasilica, 26 (1): 74-83.

Madelaine C, Pelissier R, Vincent G, Molino JF, Sabatier D, Prevost MF, de Namur C. 2007. Mortality and recruitment in a lowland tropical rain forest of French Guiana: effects of soil type and species guild. Journal of Tropical Ecology, 23(3):277-287.

Lévesque M, McLaren KP, McDonald MA. 2011. Recovery and dynamics of a primary tropical dry forest in Jamaica, 10 years after human disturbance. Forest Ecology and Management, 262:817-826.

Magurran AE. 2004. Measuring Biological Diversity, Blackwell Science, Malden, MA, USA 256 p.

Marquardt S, Marquez A, Bouillot H, Beck SG, Mayer AC, Kreuzer M, Alzérreca HA. 2009. Intensity of browsing on trees and shrubs under experimental variation of cattle stocking densities in southern Bolivia. Forest Ecology and Management, 258:1422-1428.

Marin GC, Nygard R, Rivas BG, Oden PC. 2005. Stand dynamics and basal area change in a tropical dry forest reserve in Nicaragua. Forest Ecology and Management, 208(1):63-75.

Miles L, Newton AC, DeFries RS, Ravillious C, May I, Blyth S, Kapos V, Gordon JE. 2006. A global overview of the conservation status of tropical dry forests. Journal of Biogeography, 33(3):491-505.

Oliveira-Filho AT, Carvalho WAC, Machado ELM, Higuchi P, Apolinário V, Castro GC, Silva AC, Santos RM, Borges LF, Corrêa BS, Alves JM. 2007. Dinâmica da comunidade e populações arbóreas da borda e interior de um remanescente florestal na Serra da Mantiqueira, Minas Gerais, em um intervalo de cinco anos (1999-2004). Revista Brasileira de Botânica, 30(1):149-161.

Oliveira-Filho AT, Curi N, Vilela EA, Carvalho DA. 1998. Effects of canopy gaps, topography, and soils on the distribution of woody species in a central Brazilian deciduous dry forest. Biotropica, 30(3):362-375.

Oliveira-Filho, AT, de Mello JM, Scolforo JRS. 1997. Effects of past disturbance and edges on tree community 
structure and dynamics within a fragment of tropical semideciduous forest in south-eastern Brazil over a fiveyear period (1987-1992). Plant Ecology, 131(1):45-66.

Pennigton TR, Oliveira-Filho AT, Lavin M. 2009. Woody plant diversity, evolution and ecology in the Tropics: Perspectives from seasonally dry tropical forests. The Annual Review of Ecology, Evolutiuon and Systematics, 40(1):457-467.

Phillips OL, Baker TR, Arroyo L, Higuchi N, Killeen TJ, Laurance WF, Lewis SL, Lloyd J, Malhi Y, Monteagudo A, Neill DA, Vargas PN, Silva JNM, Terborgh J, Martinez RV, Alexiades M, Almeida S, Brown S, Chave J, Comiskey JA, Czimczik CI, Di Fiore A, Erwin T, Kuebler C, Laurance SG, Nascimento HEM, Olivier J, Palacios W, Patino S, Pitman NCA, Quesada CA, Salidas M, Lezama A T, Vinceti B. 2004. Pattern and process in Amazon tree turnover, 1976-2001. Philosophical Transactions of the Royal Society of London Series B-Biological Sciences, 359:381-407.

Poorter L, Bongers L, Bongers F. 2006. Architecture of 54 moist-forest tree species: traits, trade-offs, and functional groups. Ecology, 87(5):1289-1301.

Quesada M, Sanchez-Azofeifa GA, Alvarez-Anorve M, Stoner KE, Avila-Cabadilla L, Calvo-Alvarado J, Castillo A, Espirito-Santo MM, Fagundes M, Fernandes GW, Gamon J, Lopezaraiza-Mikel M, Lawrence D, Morellato LPC, Powers JS, Neves FD, Rosas-Guerrero V, Sayago R, Sanchez-Montoya G. 2009. Succession and management of tropical dry forests in the Americas: Review and new perspectives. Forest Ecology and Management, 258:1014-1024.

Quigley MF, Platt WJ. 2003. Composition and structure of seasonally deciduous forests in the Americas. Ecological Monographs, 73(1):87-106.

Sagar R, Singh JS. 2005. Structure, diversity and regeneration of tropical dry decicuous forest of northern India. Biodiversity and Conservation, 14:935-959.

Shepherd GJ. 2006. Fitopac-Shell 1.6: Manual de usuário. Campinas, Departamento de Botânica.

Sheil D, May RM. 1996. Mortality and Recruitment Rate Evaluations in Heterogeneous Tropical Forests. Journal of Ecology, 84(1):91-100.

Silva LC, Clemente LH, Vieira MC, Nascimento ART. 2014. Regeneração natural da comunidade arbórea em um remanescente de Floresta Estacional Decidual, Uberlândia - MG. Biota, 2(5):4-22.

Souza JP, Araújo GM, Haridasan M. 2007. Influence of soil fertility on the distribution of tree species in a deciduous forest in the Triângulo Mineiro region of Brazil. Plant Ecology, 191(2):253-263.

Sukumar R, Dattaraja HS, Suresh HS, Radhakrishnan J, Vasudeva R, Nirmala S, Joshi NV. 1992. Long-term monitoring of vegetation in a tropical deciduous forest in Mudumalai, Southern India. Current Science, 62(9):608-616.

Suresh HS, Dattaraja HS, Sukumar R. 2010. Relationship between annual rainfall and tree mortality in a tropical dry forest: Results of a 19-year study at Mudumalai, southern India. Forest Ecology and Management, 
259(4):762-769.

Swaine MD, Lieberman D, Hall JB. 1990. Structure and dynamics of a tropical dry forest in Ghana. Vegetatio, $88(1): 31-51$.

Werneck MD, Franceschinelli EV. 2004. Dynamics of a dry forest fragment after the exclusion of human disturbance in southeastern Brazil. Plant Ecology, 174(2):337-346.

Werneck MS, Franceschinelli EV, Tameirão-Neto E. 2000. Mudanças na florística e estrutura de uma floresta decídua durante um período de quatro anos (1994-1998), na região do Triângulo Mineiro, MG. Revista Brasileira de Botânica, 23(4):401-413.

Venkateswaran R, Parthasarathy N. 2005. Tree population changes in a tropical dry evergreen forest of south India over a decade (1992-2002). Biodiversity and Conservation, 14:1335-1344.

Williams-Linera G, Lorea F. 2009. Tree species diversity driven by environmental and anthropogenic factors in tropical dry forest fragments of central Veracruz, Mexico. Biodiversity and Conservation, 18(12):3269-3293.

Wilkinson L. 2002. SYSTAT. The system for statistics. Illinois. 